\title{
Engineering the Micro-texture of Zn Coating by Carbon Nanotube Incorporation for Enhanced Corrosion Resistance Behavior.
}

Kompella Jyotheender ${ }^{1}$ and Chandan Srivastava ${ }^{2}$

${ }^{1}$ IISc-Bangalore, Bengaluru, Karnataka, India, ${ }^{2}$ IISc Bangalore, Bengaluru, Karnataka, India

Zinc is widely used in industries as a coating material to protect the underlying steel from corrosion [1]. Several efforts have been made to tailor the microstructure and morphology of $\mathrm{Zn}$ coatings to further enhance their corrosion resistance performance and hence the coating lifetime. One popular methodology involves incorporating micro and nano-sized foreign particles into the coating matrix [2]. It has been recently shown that incorporation of the optimum amount of carbon nanotubes (CNTs) into conventional metallic coatings significantly enhances the corrosion resistance performance of the coatings. This enhancement is due to the synergistic effect of the hydrophobicity of CNTs and the microstructural alterations caused by their incorporation into the metal matrix [3]. This report illustrates the microstructure-corrosion property correlation in electrodeposited Zn-CNT composite coatings.

Pristine Zn and Zn-CNT (CNT1 \& CNT2) composite coatings were electrodeposited from aqueous electrolyte baths containing dispersed CNTs in concentrations of $8 \mathrm{mg} / \mathrm{L} \mathrm{(Zn-CNT1)}$ and $20 \mathrm{mg} / \mathrm{L}$ (ZnCNT2). SEM micrographs suggest a compact and crack-free coating morphology (Figure 1(a)). The electrochemical impedance spectroscopy (EIS) measurements conducted in $3.5 \mathrm{wt} \% \mathrm{NaCl}$ solution indicated the highest corrosion resistance for CNT1 coatings followed by pristine Zn and Zn-CNT2 coating. The Polarization resistance $\left(\mathrm{R}_{\mathrm{p}}\right)$ values obtained were $1197.9 \Omega-\mathrm{cm}^{2}, 1559.4 \Omega-\mathrm{cm}^{2}$, and 382.4 $\Omega$-cm ${ }^{2}$ for pristine Zn, Zn-CNT1, and Zn-CNT2 coatings, respectively.

Orientation distribution maps were obtained from the coating cross-section using the electron backscatter diffraction (EBSD) technique (Figure 1(b)). Pristine Zn and Zn-CNT1 coatings showed strong basal plane texture along the growth direction. Whereas Zn-CNT2 coating indicated planes with $\{4\}$ orientation (Figure 1(c)). Grain boundary character distribution (GBCD) suggested the presence of a higher fraction of low angle grain boundaries (LAGBs) in Zn-CNT composite coating compared to pristine Zn coating (Figure 2(a)). Special grain boundaries in $\mathrm{Zn}$ are possible at some well-defined angle/axis misorientations. The energy of boundaries (increasing order) along [2-1-10] axis are as follows: $65^{\circ} /[[2-1-10]]<77^{\circ} /[2-$ $1-10]<36^{\circ} /[2-1-10]<47^{\circ} /[2-1-10]$; along [10-10] axis are as follows: $75^{\circ} /[10-10]<62^{\circ} /[10-10]<$ $32 \%[10-10]<43 \% /[10-10]$ [4,5]. Pristine Zn coating did not show any special boundaries (Figure 2(b)). Whereas Zn-CNT1 coating exhibited a higher fraction of special boundaries than Zn-CNT2, suggesting that the Zn-CNT1 coating had a large fraction of high angle grain boundaries (HAGBs) with lower energies when compared to Zn-CNT2 coating.

The results indicated that the incorporation of CNTs leads to changes in the Zn-CNT composite coatings micro-texture. Zn-CNT1 coating exhibited the highest corrosion resistance due to inert grains with a basal plane texture that resisted trans-granular corrosion and a high fraction of low energy grain boundaries (LAGBs and special boundaries), which resisted the intergranular corrosion. Pristine Zn coating had only grains with inert texture, and Zn-CNT2 coating had only LAGBs and some special boundaries. Therefore, higher intergranular corrosion in pristine $\mathrm{Zn}$ coating and bulk corrosion in Zn-CNT2 coating led to higher corrosion rates in these cases. 

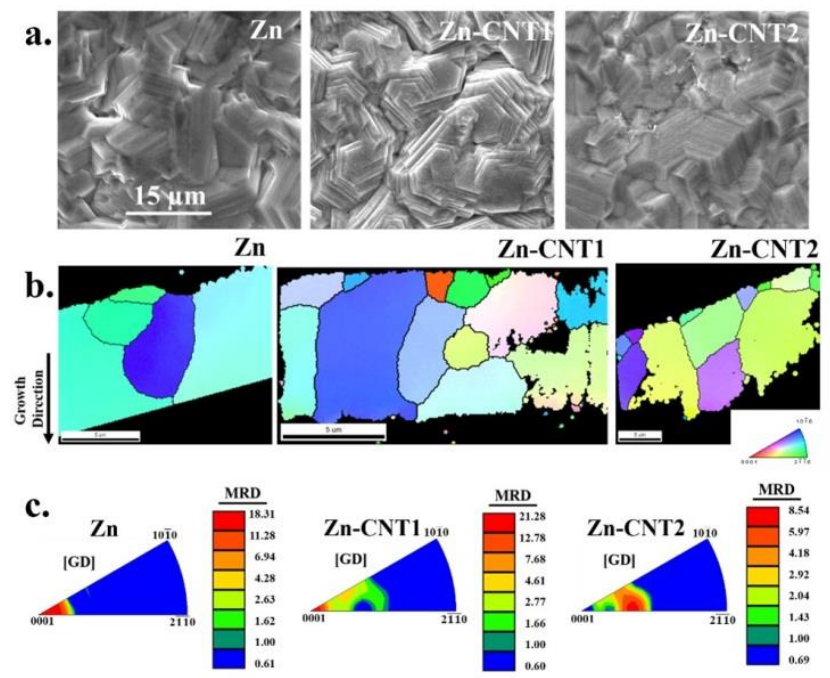

Figure 1. Figure 1. (a) SEM micrographs representing the surface morphology, (b) orientation distribution maps representing the coating cross-section, and (c) Inverse pole figures (IPFs) of along growth direction in $\mathrm{Zn}$ and $\mathrm{Zn}-\mathrm{CNT}$ coatings.
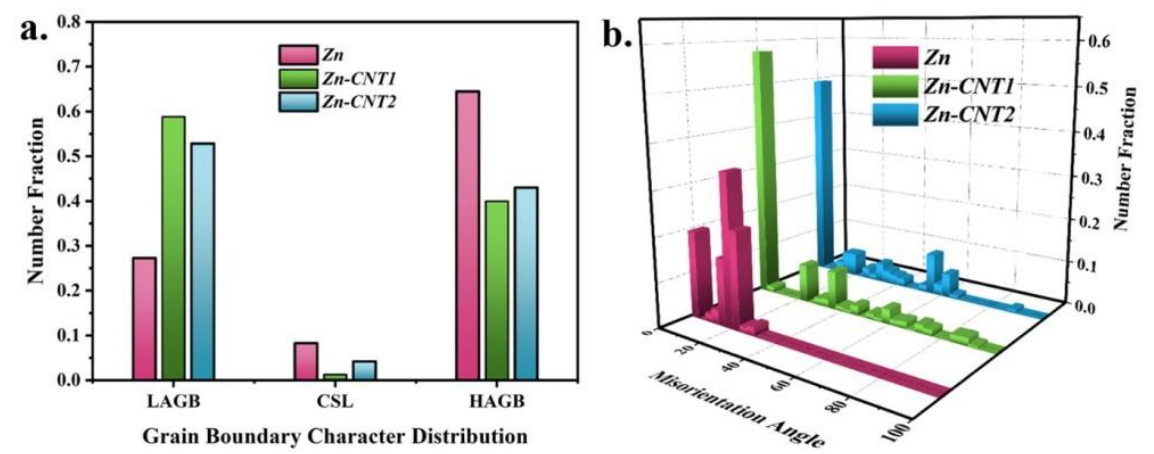

Figure 2. Figure 2. (a) Grain boundary character distribution, (b) Misorientation angle distribution in $\mathrm{Zn}$ and Zn-CNT coatings.

\section{References}

[1] G.D. Wilcox and D.R. Gabe, Corros. Sci. 35 (1993) p. 1251-1258.

[2] P.I. Nemes, et al. Surf. Coatings Technol. 252 (2014) p. 102-107.

[3] M.K. Punith Kumar, M.Y. Rekha, C. Srivastava, Corros. Rev. 39(1) (2021) p. 15-26.

[4] J. Wang, I.J. Beyerlein, Metall. Mater. Trans. A Phys. Metall. Mater. Sci. 43 (2012) p.3556-3569

[5] J. Wang, I.J. Beyerlein, Model. Simul. Mater. Sci. Eng. 20 (2012) p. 01-22. 